\title{
Numerical Estimation of the Critical Reynolds Number for Flow Past one Square Cylinder with Symmetric Geometry Boundary Condition
}

\author{
X. H. Wangi*, W. F. Zhu' ${ }^{2}$, Z. Y. $\mathrm{He}^{3}$ \\ ${ }^{1}$ Institute of Fluid Engineering, College of Mechanical and Energy Engineering, Zhejiang University, \\ Hangzhou 310027, China \\ ${ }^{2}$ Zhejiang University of Science and Technology, Hangzhou 310012, China \\ ${ }^{3}$ School of Municipal \& Environmental Engineering, Harbin Institute of Technology, Harbin 150090, \\ China \\ Email: tommewang@yahoo.com.cn
}

\begin{abstract}
It is well known that the steady flow past a circular cylinder loses stability at Re takes the value about 50 (Y. Ding et al, 1999). Most papers about the characterization and understanding of the stability for the flow past blunt bodies are mainly carried out for such flow past one circular cylinder. And there is a large variation in the values of $R e_{c r}$ and correspondingly the values of $S t_{c r}$ reported by different reseachers. Bhascar and Sunjay (2006) have attributed it to the effect of blockage (here, it means the ratio of the diameter of cylinder to the lateral width of domain). And for high $R e_{c r}$ first decreases and then increases with the increase of the blockage. And the correspondingly values of $S t_{c r}$ are quite sensitive to the blockage. In this paper, we attampt to estimate the critical Re for the flow past one square cylinder. It is obviously that geometry symmetry or attack degree will not change just with rotation of the circular cylinder, but for the square cylinder, the symmetry or the degree will not keep for the rotation. So the numerical estimation of the critical Re for the flow past square cylinders should be carried out for the symmetric or unsymmetric geometry boundary conditions separately. Based on the calculation of the lid driven cavity flow at $R e=100$ and 1000 , a second order Euler-Taylor-Galerkin finite element method was used to estimate the critical Reynolds number for flow past one square cylinder with zero attact degree through direct time integration of the NS equationes. The role of blockage on such flow was analysed at $R e=100$. It was found that the averged St tend to be constant as blockage took the value larger than 50. The critical Reynolds number is then computed. As the result shown, it was estimated that $R e_{C r}=40.11$. And the computation for unsymmetric geometry condition will discussed laterly.
\end{abstract}

\section{REFERENCES}

1. Ding Y., Kawahara M. Three dimensional linear stability analysis of incompressible viscous flows using the finite element method Int. J. Numer Meth. Fluids, 1999;31:451-479

2. Kumar B., Mittal S. Prediction of the critical Reynolds number for flow past a circular cylinde, Comput. Methods Appl. Mech. Engrg., 2006;195:6046-6058 